\title{
Pre-SMA actively engages in conflict processing in human: A combined study of epicortical ERPs and direct cortical stimulation.
}

\section{$\operatorname{AUTHOR}(S)$ :}

Usami, Kiyohide; Matsumoto, Riki; Kunieda, Takeharu; Shimotake, Akihiro; Matsuhashi, Masao; Miyamoto, Susumu; Fukuyama, Hidenao; Takahashi, Ryosuke; Ikeda, Akio

\section{CITATION:}

Usami, Kiyohide ...[et al]. Pre-SMA actively engages in conflict processing in human: A combined study of epicortical ERPs and direct cortical stimulation.. Neuropsychologia 2013, 51(5): 1011-1017

\section{ISSUE DATE:}

2013-04

URL:

http://hdl.handle.net/2433/174131

\section{RIGHT:}

@ 2013 Elsevier Ltd.; この論文は出版社版でありません。引用の際には 出版社版をご確認ご利用ください。; This is not the published version. Please cite only the published version. 


\section{Pre-SMA actively engages in conflict processing in human: a combined study of epicortical ERPs and direct cortical stimulation}

\section{Authors:}

Kiyohide Usamia, Riki Matsumotoa,*, Takeharu Kuniedab ${ }^{a}$, Akihiro

Shimotake ${ }^{a}$, Masao Matsuhashic, Susumu Miyamotob, Hidenao Fukuyamac, Ryosuke Takahashia , Akio Ikeda ${ }^{a},{ }^{*}$

aDepartment of Neurology, Kyoto University Graduate School of Medicine, 54 Kawahara-cho, Shogoin, Sakyo-ku, Kyoto, 606-8507, Japan;

bDepartment of Neurosurgery, Kyoto University Graduate School of Medicine, 54 Kawahara-cho, Shogoin, Sakyo-ku, Kyoto, 606-8507, Japan; cHuman Brain Research Center, Kyoto University Graduate School of Medicine, 54 Kawahara-cho, Shogoin, Sakyo-ku, Kyoto, 606-8507, Japan

*To whom correspondence should be addressed.
Akio Ikeda, M.D., Ph.D. and Riki Matsumoto, M.D., Ph.D.
Department of Neurology, Kyoto University Graduate School of Medicine, 54 Kawahara-cho, Shogoin, Sakyo-ku, Kyoto, 606-8507, Japan
Phone number: $\quad$ +81757513772
Fax number: $\quad$ +81757519416
E-mail: akio@kuhp.kyoto-u.ac.jp (A.I) matsumot@kuhp.kyoto-u.ac.jp (R.M.)

Key words:

pre-SMA; conflict processing; medial frontal cortex; direct cortical stimulation; event-related potential 


\section{Abstract}

Previous non-invasive studies have proposed that the deeply seated region of the medial frontal cortex engages in conflict processing in humans, but its core region has remained to be elucidated. By means of direct cortical stimulation, which excels other techniques in temporal and spatial resolutions and in the capacity of producing transient, functional impairment even in the deeply located cortices, we attempted to obtain direct evidence that the pre-supplementary motor area (pre-SMA) actively engages in conflict processing. Subject was a patient with right frontal lobe epilepsy who underwent invasive presurgical evaluation with subdural electrodes placed on the medial and lateral frontal cortices. During a conflict task modified Eriksen flanker task, direct cortical stimulation was delivered time-locked to the task at the inferior part of the medial superior frontal gyrus (inferior medial SFG), the superior part of the medial SFG, and the middle frontal gyrus. By adopting the session of sham stimulation that was employed as a within-block control, event-related potentials (ERPs) were recorded from the medial and lateral frontal cortices. The inferior medial SFG showed a significant ERP difference between trials with more and less 
conflict, while the other frontal cortices did not. Among the 3 stimulus sites, only stimulation of the inferior medial SFG significantly prolonged reaction time in trials with more conflict. Anatomically, the inferior medial SFG corresponded with the pre-SMA (Brodmann area 8). It was located 1-2 cm rostral to the vertical anterior commissure line where cortical stimulation elicited arrest of motion (the supplementary negative motor area). Functionally, this area corresponded to the dorso-rostral portion of the activation loci in previous neuroimaging studies focusing on conflict processing. By combining epicortical ERP recording and direct cortical stimulation in a human brain, this study, for the first time, presented one direct piece of evidence that the pre-SMA actively participates in conflict processing. 


\section{Highlights}

- We assigned a conflict task to an epilepsy patient undergoing presurgical

evaluation with subdural electrodes.

- Degree of conflict modulated ERPs during the task at a deeper portion of the pre-supplementary motor area.

- Electrical cortical stimulation of this restricted area prolonged reaction time in trials with more conflict.

- The stimulation site was located 1-2 $\mathrm{cm}$ rostral to the supplementary negative motor area. 


\section{Introduction}

In daily life as we drive a car or play tennis, we repeatedly and immediately select, presumably appropriate, one choice, facing upon different kinds of conflict. Processing such conflict, namely, conflict processing is one of the most important aspects of the cognitive control for behavior. The conflict monitoring theory, which was introduced by Carter et al. (1998) and later developed by Botvinick et al. (2001), is a representative. According to this theory, we, humans, possess an ability of monitoring response conflict, which emerges after multiple response programs start running simultaneously. By accumulating evidence with various modalities, Botvinick et al. proposed that the dorsal anterior cingulate cortex (ACC) [Brodmann Area (BA) 32] in the medial frontal cortex (MFC) is pivotal for monitoring response conflict (Botvinick, Braver, Barch, Carter, \& Cohen, 2001; Botvinick, Cohen, \& Carter, 2004; Cole, Yeung, Freiwald, \& Botvinick, 2009). On the other hand, other researchers analyzed conflict before response and error after response separately in functional MRI (fMRI) activation studies. They argued that the ACC mainly processes error after response while the more dorsal area, namely, the medial superior frontal gyrus (SFG) including the 
pre-supplementary motor area (pre-SMA) [medial BA 8 or 6] manages conflict processing, although the activation areas for the two functions indeed overlap to a considerable degree (Garavan, Ross, Murphy, Roche, \& Stein, 2002; Kiehl, Liddle, \& Hopfinger, 2000; Ridderinkhof, Ullsperger, Crone, \& Nieuwenhuis, 2004; Rushworth, Walton, Kennerley, \& Bannerman, 2004; Ullsperger \& von Cramon, 2001, 2003). Some also proposed that the ACC is engaged when emotional information must be ignored or controlled (Banich, et al., 2009), or that it mediates ongoing behavioral adaptation (Sheth, et al., 2012). Through the extensive debate in the last decade as to the core region for conflict processing (Cole, et al., 2009; Nachev, Kennard, \& Husain, 2008; Rushworth, et al., 2004), a key structure for this particular function seems to reside relatively deep (not superficial) in the MFC.

These findings have been essentially obtained by non-invasive studies in different modalities, i.e., lesion studies (Fellows \& Farah, 2005; Floden \& Stuss, 2006; Verfaellie \& Heilman, 1987), fMRI activation studies (Barch, et al., 2001; Milham \& Banich, 2005; Ridderinkhof, et al., 2004), and event-related potential (ERP) studies (van Veen \& Carter, 2002a). Each non-invasive technique, however, has some methodological limitations; the 
former is of the degree of plasticity of brain function after lesion formation and the latter two of indirect representation of neural function. In contrast to these studies, intervention or stimulation studies can delineate the cortices necessary for a particular function by producing transient, functional impairment, and therefore complement these non-invasive techniques. For example, when transcranial magnetic stimulation (TMS) was applied to the MFC during tasks that contain more conflict in healthy subjects, their performance was disrupted with higher error rate or prolonged reaction time (Chen, Muggleton, Tzeng, Hung, \& Juan, 2009; Taylor, Nobre, \& Rushworth, 2007). It is no doubt that TMS is a valuable non-invasive intervention tool, but, the stimulation effects are usually confined to the superficial cortices within $3 \mathrm{~cm}$ from the scalp (Rossi, Hallett, Rossini, \& Pascual-Leone, 2009). In contrast to TMS, direct cortical stimulation can assess deeply seated cortices, such as those in the medial wall of the hemisphere, with good spatial resolution $(\sim 1 \mathrm{~cm})$. It has been and is still currently a gold standard measure to map eloquent cortical areas in the field of functional neurosurgery. Although it is available only for patients who undergo invasive presurgical evaluation with intracranial electrodes before epilepsy surgery, it 
provides us with the rare opportunity to investigate the MFC by epicortical ERP recording and direct cortical stimulation. It potentially helps solve the ongoing concerns about the cortical region responsible for conflict processing in the MFC.

We here present a patient in whom we successfully recorded ERPs and stimulated both the superficial and deeper portions of the pre-SMA during a task that imposes conflict for selecting correct answers - a modified version of the Eriksen flanker task (Eriksen \& Eriksen, 1974). We applied cortical stimulation time-locked to the task so that we could evaluate its functional interference in the pre-SMA with reaction time (RT) and accuracy. We hypothesized that a different degree of conflict modulates ERPs in the pre-SMA, and that direct cortical stimulation to the area of ERP modulation prolongs RT or impairs accuracy during the task. By means of these methods, we attempted to obtain direct evidence that the pre-SMA actively engages in conflict processing.

\section{Materials and methods}

\subsection{Patient}


The subject was a 44-year-old, right-handed man with medically intractable right frontal lobe epilepsy. The seizures consisted of head version to the left and asymmetric bilateral tonic posturing with preserved consciousness. Interictally he had normal motor functions but a mild cognitive dysfunction (total IQ 65 in WAIS-R). MRI revealed a tumor (oligoastrocytoma) in the right SMA. We suspected that the epileptic focus was located around the tumor according to the non-invasive presurgical evaluations. The patient finally underwent chronic implantation of subdural electrodes on the medial and lateral cortical surfaces of the right hemisphere in order to locate the epileptic focus and map cortical functions (Fig. 1a). The patient gave written informed consent to the protocol approved by the Ethics Committee of our institute (No.C533).

\subsection{Anatomical localization of electrodes in the individual and standard} space

We used a magnetization-prepared rapid gradient echo (MPRAGE) sequence for anatomical T1-weighted volume data acquisition. MPRAGE volumetric scan was taken before surgery, and also after implantation when 
subdural electrodes were in place. As for the obtained scan image after implantation, we located each electrode by confirming its signal void due to the property of platinum alloy (Matsumoto, et al., 2004). In order to compare the present findings with non-invasive fMRI studies, electrodes were non-linearly co-registered to the scan image taken before implantation, and then to MNI standard space (ICBM-152) using FNIRT (www.fmrib.ox.ac.uk/fsl/fnirt) (Matsumoto, et al., 2011). Anatomical labels for electrodes in the MNI standard space were determined in reference to the atlas of Talairach Daemon implemented in FSL View (Lancaster, et al., 2000). For the purpose of 3D display in figures, the grey matter segmentation was done for MPRAGE taken before implantation by Freesurfer software (http://surfer.nmr.mgh.harvard.edu/) and presented in FSL View (www.fmrib.ox.ac.uk/fsl/fslview) (Fig. 1a).

\subsection{Direct electrical cortical stimulation and functional cortical mapping}

Direct electrical cortical stimulation was performed with subdural electrodes (platinum-made, inter-electrode distance of $1 \mathrm{~cm}$, recording surface diameter of $2.3 \mathrm{~mm}, \mathrm{AD}-\mathrm{TECH}, \mathrm{WI})$. Repetitive square-wave 
currents of alternating polarity with a pulse width of $0.3 \mathrm{~ms}$ and a frequency of $50 \mathrm{~Hz}$ were delivered for 1-5 s to the cortex through a pair of adjacently placed electrodes. Intensity was gradually increased from 1 to $15 \mathrm{~mA}$ until positive motor responses, e.g., muscle twitch or tonic posturing, appeared. Only the trials without afterdischarges were evaluated. When positive symptoms were not elicited, negative motor responses were also examined by having the patient perform rapid alternating movements in the tongue, hands and feet $(10-15 \mathrm{~mA}, 5 \mathrm{sec})$. Once he was unable to continue those movements while awake during stimulation, namely negative motor responses were elicited, the stimulated area was labeled as the negative motor area (NMA). Two NMAs were known so far, one around the ventral premotor area (primary NMA), and the other anterior to the SMA around the vertical anterior commissure (VAC) line (supplementary NMA) (Lüders, Dinner, Morris, Wyllie, \& Comair, 1995). The stimulation method has been reported elsewhere (Matsumoto, et al., 2007).

In this patient, functional cortical mapping successfully localized the supplementary NMA at the border between the pre-SMA and the SMA proper as reported elsewhere (Ikeda, et al., 1999) [the MNI coordinates (x, y, 
z) of $(4,4,56)$ and $(4,14,58)]$. The epileptic focus was identified at and around the tumor located in the SMA (Fig. 1a). In the right MFC rostral to the NMA, electrical cortical stimulation did not elicit any positive or negative motor responses.

\subsection{Modified Eriksen flanker task}

The Eriksen flanker task (Eriksen \& Eriksen, 1974) is frequently used for studying conflict processing. We employed a modified version of the task. The patient was told to report as quickly as possible the direction (right or left) of an arrow in the center among five arrows lined horizontally.

Namely, the arrow was located between two flankers (distractor arrows) on each side. Then, two congruent (“<<<<<” and " $>>>>>$ ") and two incongruent (">><>> and " $<<><<")$ signals (herein each is termed as a flanker-signal) were employed. Each flanker-signal was presented pseudorandomly with equal probability (25\%). We chose the hand contralateral to the side of implantation, namely, the left hand, for the response. The patient responded by pressing the right button with the left index finger when the central arrow was directed to the right, and by the left button with the left middle 
finger when directed to the left. Previous studies have reported that RT was longer in incongruent trials than in congruent trials (Botvinick, et al., 2004; Eriksen, 1974). It is because the distractors make more conflict in incongruent trials so that subjects need to inhibit "prepotent responses" to select a real answer.

\subsection{Study design}

The patient watched an LCD display $1.0 \mathrm{~m}$ in front, sitting comfortably in Fowler's position on the bed. The visual angle of a flanker-signal (one arrow and two flanker arrows on each side) was $3.9^{\circ}$ wide and $0.65^{\circ}$ tall. In one trial, a circle appeared first in the center of the display for $1 \mathrm{~s}$, and was then replaced by one of the flanker-signals for $1 \mathrm{~s}$ (Fig. 1b). The patient was told not to blink at all while the circle and the flanker-signal appeared. Inter-trial interval was $4 \mathrm{~s}$ and the patient should look at the cross in the center. Before the implantation surgery, we confirmed by rehearsal that the patient could appropriately perform the task.

The study was performed three days before the focus resection surgery, after clinical evaluations were essentially complete. Because of the 
limited opportunity allocated for this research protocol, we could not perform the paradigm twice in separate days: one for ERP recording and the other for intervention by direct cortical stimulation. Instead, we performed the paradigm with and without stimulation in the same day. By adopting the sessions without stimulation (sham stimulation), we could obtain ERPs during the modified flanker task. Furthermore, a comparison between the sessions with and without stimulation, i.e. employing a within-block control (sham stimulation), enabled us to analyze the effect of intervention.

\subsection{Intervention during the modified flanker task}

Direct cortical stimulation $(50 \mathrm{~Hz}$, pulse width of $0.3 \mathrm{~ms}$, alternating polarity) was delivered separately in a block fashion for three locations - the inferior part of the medial superior frontal gyrus (SFG) (inferior medial SFG: BA 8) [site 1: the MNI coordinates $(\mathrm{x}, \mathrm{y}, \mathrm{z})$ of $(4,24,48)$ and $(4,34,50)$, the superior part of the medial SFG (superior medial SFG: BA 6) [site 2: (4, 24, 58) and $(4,34,60)]$, and the middle frontal gyrus (MFG: BA 6) in the lateral frontal cortex [site 3: $(34,10,64)$ and $(38,18,58)]$ (Fig. 1a). In the medial cortex, we selected the inferior and superior medial SFG pairs for 
stimulation sites, since 1) they were rostral to the VAC line, 2) we attempted to investigate a possible functional difference along the dorso-ventral axis (deep vs. superficial) and 3) we avoided the supplementary NMA where stimulation stopped the motor task itself due to negative motor responses. The lateral stimulus site was chosen at the MFG because it was the closest to the medial stimulus sites. All the stimulated electrodes were away from the epileptic focus that was identified at and around the tumor in the SMA.

The experimental blocks started with the inferior medial SFG block followed by the superior medial SFG and the MFG blocks. In each block, we first confirmed that electrical stimulation did not produce any positive or negative motor responses and afterdischarges in the condition used for intervention (5 mA, 1 s) (Electrical stimulator SEN-7203, Nihon Koden, Tokyo, Japan). We then performed two sessions of the modified flanker task - the first session (48 trials) without stimulation (sham stimulation) and the second session (48 trials) with stimulation. In each session, the number of trials was controlled among the four different flanker-signals [12 trials each for two congruent (" $<<<<<", ">>>>>$ ") and two incongruent (" $>><>>$ ", "<<><<") signals]. The stimulation was delivered from the onset of display of 
a flanker-signal for $1 \mathrm{~s}$, namely during the whole presentation of the flanker-signal (Fig. 1b). We produced the sounds of the relay switch needed for stimulus delivery also in sham sessions so that the subject could not know whether the session was with or without stimulation.

We confirmed that no seizures or no afterdischarges occurred throughout the experiment by carefully monitoring electrocorticogram (ECoG). ECoG, electrooculogram, and all the related signals such as the timing for visual stimuli and responses of button-press were digitally recorded and stored on hard disc in the recording system (EEG1000, Nihon Koden, Tokyo, Japan). The data were sampled at 2,000 Hz with a band-pass filter of $0.08-600 \mathrm{~Hz}$. Signals from the subdural electrodes were referenced to a scalp electrode placed on the left mastoid process.

Among all the trials $[\mathrm{n}=48$ trials $\times 2$ sessions (stimulation, sham) $\times$ 3 stimulus sites], 3 trials were rejected for analysis of RT. An error occurred in 1 congruent trial during the inferior medial SFG stimulation and eye blinks occurred during $-50 \mathrm{~ms}$ to $+50 \mathrm{~ms}$ from the onset of the flanker-signal presentation in 2 trials (1 incongruent trial during the superior medial SFG stimulation, and 1 congruent trials during the MFG sham stimulation) 
(Supplementary Table).

\subsection{Analysis of ERPS}

By employing all sham sessions (congruent trials: $\mathrm{n}=71$, incongruent trials:

$n=72$ ), we averaged ECoGs in an off-line manner to obtain ERPs (Matlab

scripts, custom-made in Matlab version 2010a) for congruent and

incongruent trials separately. In sham sessions, ECoGs were also recorded

from the electrodes used for delivering stimulus. ECoGs were averaged

time-locked to the onset of the warning signal (screen of a circle in the

center). A total of $4 \mathrm{~s}$ was set as the whole time window $(-1$ to $+3 \mathrm{~s}$ from the

onset of the warning signal). The initial $1 \mathrm{~s}$ of the analysis window was set as

the baseline for measurement. In order to analyze the ERP difference during

presentation of the flanker-signal, raw ECoG data were filtered with a

band-pass of 0.08-10 Hz, and then the ECoGs during the flanker-signal

presentation (a total of 2,001 time points at 1-2 s from the fiducial point)

were selected for further statistical analyses. Initially, two-tailed two sample

$t$ test (degree of freedom: 141) was performed to calculate $t$-value for every

time sample during a period of flanker-signal presentation. To solve the 
multiple comparison problem arising from multiple time-point sampling and multiple recording site, we then applied a nonparametric statistical testing (Maris \& Oostenveld, 2007). We first, selected all the samples where $t$-value satisfied $p<0.05$, and clustered them in connected sets on temporal adjacency at each electrode independently. By adopting only clusters having durations longer than $20 \mathrm{~ms}$, we then calculated the sum of the $t$-values within each cluster and made cluster-level statistics. Nonparametric statistics were calculated using a permutation test by our custom script in Matlab. The congruent $(n=71)$ and incongruent $(n=72)$ trials were collected in a single set, and the set was randomly re-partitioned into two groups of trials of $n=71$ and $n=72$. Comparing the two groups by aforementioned two-tailed $t$ test, cluster-level statistics were calculated. Then only the highest statistic from all of the recording electrodes was taken. The procedure was repeated 5,000 times and the null distribution of the maximum cluster-level statistics was compared to the original cluster-level statistics. A $p$-value threshold was defined $<0.05$.

\subsection{Analysis of behavior}


The main dependent variable was RT. Only RT of trials with correct answer was analyzed. RT was defined as the time difference from the onset of a flanker-signal to a button press (Fig. 1b). First, 3-way ANOVA [condition (congruent; incongruent) $\times$ stimulation $($ sham stimulation; stimulation $) \times$ location (inferior medial SFG; superior medial SFG; MFG)] was performed to evaluate the overall performance and to confirm the difference of performance between congruent and incongruent trials. A $p$-value threshold was defined $<0.05$. Second, RT was analyzed for each location. After separating congruent and incongruent trials, we compared RT in the sham session with that in the stimulation session to test the hypothesis that direct cortical stimulation impairs performance. A $p$-value threshold was defined < 0.025 after Bonferroni correction. In order to evaluate a possible effect of the block order upon RT as a confounding factor, one-way ANOVA across the three locations (inferior medial SFG; superior medial SFG; MFG) was also performed for sham sessions in each condition (congruent; incongruent). A $p$-value threshold was defined $<0.05$.

\section{Results}




\subsection{ERP during Conflict Processing}

By adopting the sessions with sham stimulation, ERPs during execution of the modified flanker task were compared between the congruent $(n=71)$ and incongruent $(\mathrm{n}=72)$ trials. A significant amplitude difference was observed between the waveforms of the two conditions (congruent vs. incongruent) only at a single electrode in the inferior medial SFG stimulus site (see an electrode with an asterisk in Fig. 2). The difference appeared from $350 \mathrm{~ms}$ after the onset of the flanker-signal and lasted for $536 \mathrm{~ms}[p=0.02$, nonparametric permutation test (cluster-level statistics)]. No significant differences were noted in ERPs at the NMA and at the other electrodes $(p>$ $0.05)$.

\subsection{Intervention to Conflict Processing}

Irrespective of the condition (congruent vs. incongruent), stimulation at the three locations did not produce any subjective symptoms including urge to move. Three-way ANOVA [condition (congruent; incongruent) $\times$ stimulation (sham stimulation; stimulation) $\times$ location (inferior medial SFG; superior medial SFG; MFG)] showed that the main effects were significant at 
condition $[F(1,273)=59.802, p<0.001]$, stimulation $[F(1,273)=4.245, p=$ 0.040], and location $[F(2,273)=3.65, p=0.027]$. The main effect at condition, i.e., longer $\mathrm{RT}$ in incongruent than congruent trials, was consistent with previous reports using flanker tasks. Besides the main effects at stimulation and at location, the significant interaction was observed between stimulation and location $[F(2,273)=3.373, p=0.036]$, indicating that intervention or stimulation effects to RT differed depending on the location of stimulation.

After confirming the interaction between stimulation and location, RT was then analyzed for each location using two-tailed two sample $t$ test. In the incongruent trials of the inferior medial SFG block, RT was significantly longer in the stimulation session than in the sham session $[797 \pm 59 \mathrm{~ms}$ vs. $711 \pm 40 \mathrm{~ms}, t(46)=2.494, p=0.016$ ] (Fig. 3). In congruent trials of the same block, the difference was not significant $[658 \pm 51 \mathrm{~ms}$ vs. $600 \pm 66 \mathrm{~ms}, t(45)=$ 1.435, $p=0.158$ ]. In the other two blocks (superior medial SFG and MFG), RT was not significantly different between the sham and stimulation sessions in any trials [congruent trials $(p=0.322)$ and incongruent trials $(p=$ 0.153) in the superior medial SFG block, congruent trials $(p=0.884)$ and incongruent trials $(p=0.999)$ in the MFG block]. One-way ANOVA across the 
three locations (inferior medial SFG; superior medial SFG; MFG) for sham sessions in each condition (congruent; incongruent) showed that location, namely, the block order did not have any significant effect on RT both in congruent trials $[F(2,68)=0.727, p=0.487]$ and in incongruent trials $[F(2$, 69) $=0.275, p=0.761]$.

Since the error occurred only once $(\mathrm{RT}=888.5 \mathrm{~ms})$ in congruent trials during the inferior medial SFG block, we did not further analyze the error rate. All the behavioral data can be found in Supplementary Table.

\subsection{Comparison with non-invasive fMRI findings}

For comparison with activation loci of fMRI studies focusing on conflict processing (Barch, et al., 2001; Braver, Barch, Gray, Molfese, \& Snyder, 2001; Carter, et al., 1998; MacDonald, Cohen, Stenger, \& Carter, 2000; Milham \& Banich, 2005; Milham, et al., 2001; Ullsperger \& von Cramon, 2001, 2003), the electrodes at the inferior medial SFG and NMA were shown in the MNI standard space (Fig. 4). The inferior medial SFG electrodes were located in the most dorsal and rostral portion of the activation areas in the MFC. 


\section{Discussion}

Direct cortical stimulation was applied time-locked to a modified version of the Eriksen flanker task in a patient with right frontal lobe epilepsy. By adopting the sessions with sham stimulation, epicortical ERPs were also obtained during the same task. The inferior medial SFG showed a significant ERP difference between the congruent and incongruent trials. Among the 3 stimulus sites, it was only this inferior medial SFG that, upon stimulation, showed significantly prolonged $\mathrm{RT}$ in the incongruent trials. This area was anatomically and functionally different from the NMA where cortical stimulation elicited so-called negative motor response. Taking the ERP and intervention findings into account together, we concluded that the pre-SMA, BA 8 actively engaged in information processing during a task that evokes conflict in the present patient. It was not the ACC, which has been thought to be a key structure for conflict monitoring.

Scalp-recorded ERPs during the Eriksen flanker task shows N200, a frontocentral midline negativity that peaks at $\sim 200 \mathrm{~ms}$ after the flanker-signal onset. Since N200 amplitude is higher in incongruent trials 
than in congruent trials (Bartholow, et al., 2005; Kopp, Rist, \& Mattler, 1996), N200 is considered as an ERP correlate of conflict that this task evokes.

Dipole source modeling indicated its major source at the ACC (BA 32) (Van Veen \& Carter, 2002b). In contrast, the statistically significant difference of ERP amplitude was observed between the two conditions from 350 to $886 \mathrm{~ms}$ from the flanker-signal onset in the present patient (Fig. 2). The polarity was positive at the inferior medial SFG, i.e., more positive ERP in the incongruent trials than in the congruent trials. Difference in the latency and polarity between the present study and scalp-recorded ERP studies could be mainly due to the special conditions in the present study such as 1) epicortical recording by subdural electrodes and 2) the limited extent of electrode coverage. Since the subdural electrodes usually record restricted local field potentials just beneath the electrodes, the electrodes on the medial surface reflected neural activities mainly vertical to the interhemispheric fissure (namely tangential to the scalp vertex) in the present patient. Therefore, the polarity was not necessarily identical to that recorded at the scalp vertex. Indeed, an invasive ERP study during a flanker task revealed P3-like positive potentials at 250-600 ms after the onset of incongruent 
signals in the cingulate gyrus (Rusnáková, Daniel, Chladek, Jurák, \& Rektor, 2011). Regarding the latency, if we adopt the statistical significance at each sampling-time (i.e., two sample $t$ test without cluster-level statistics, see black bars in Fig. 3), both the inferior and superior medial SFG showed earlier ERP differences $(<250 \mathrm{~ms}$ ) with similar morphology (more positive in the incongruent task). Although the ACC (BA 32) was not covered by the electrodes in the present patient, these earlier activities, together with those at the ACC, may constitute a part of N200 components.

Among the three stimulation sites, only stimulation of the inferior medial SFG significantly prolonged RT during the Eriksen flanker task. Converging evidence of both intervention and ERP suggests that the ERP difference at the inferior medial SFG is not only an epiphenomenon but a neural correlate of conflict processing. While stimulation significantly prolonged $\mathrm{RT}$ in the incongruent trials, only a tendency $(\mathrm{p}=0.158)$ was observed in congruent trials. It does not necessarily imply that no conflict occurred in congruent trials, but indicates that the degree of conflict was lower in the congruent trials if stimulation influenced RT according to the degree of conflict (Yeung, Botvinick, \& Cohen, 2004). In other words, the 
stimulation effect depended on how largely conflict was loaded in each trial.

In the present study, no remarkable changes were found in the error rate (0/144 trials with sham stimulation vs. $1 / 144$ with stimulation), while $\mathrm{RT}$ prolonged in incongruent trials when cortical stimulation was applied to the inferior medial SFG. On the other hand, microstimulation in monkey and TMS in human studies reported that stimulation changed the error rate in conflict tasks (Chen, et al., 2009; Isoda \& Hikosaka, 2007; Taylor, et al., 2007). The absence of substantial errors in the present study could be explained by the study design we employed to avoid the patient's dropout the longer display ( $1 \mathrm{~s})$ of the flanker-signal and the low intensity of stimulation (5 mA).

As compared with the activation loci of fMRI studies for conflict processing, the location of the inferior medial SFG in the present patient was situated in the most dorsal and rostral portion of these loci. Dorso-rostral deviation might be based on either the different modality of investigation or the limited extent of electrode coverage in the medial cortices. The inferior medial SFG was located immediately rostral to the NMA where neither similar waveform nor significant ERP difference was observed. The NMA, 
which usually resides around the boundary between the pre-SMA and SMA

proper, is often described in terms of response inhibition in behavioral control (Nachev, et al., 2008; Sharp, et al., 2010; Simmonds, Pekar, \& Mostofsky, 2008). Although response inhibition was not precisely studied with a proper task such as Go/No-Go task, aforementioned anatomical relationship implies that conflict was processed in the inferior medial SFG, (the pre-SMA, BA 8) whereas response inhibition was processed in the more caudal NMA in this particular patient.

The present study has several limitations. First, the specific role of the inferior medial SFG (the pre-SMA, BA 8) in the Eriksen flanker task remains elusive. Although both stimulation effect and ERP difference converged at the inferior medial SFG during the display of the flanker-signal, the findings themselves could not specify its role to one phase of conflict processing that involves the processing of the perceptual conflict (or merely the stimulus difference) between the flankers and the central items, response conflict, and response programming. These results indicate that the area was involved in the task, at some point in the processing stream. Further data accumulation using other tasks, such as the Stroop task, in the 
invasive setting would clarify the phase where the pre-SMA actively engages.

Secondly, the study design was not optimal because of the time constraints due to clinical needs. To complete all the examinations without patient's dropout, we first stimulated the inferior medial SFG, and then other two sites. Although we were able to employ within-block controls (sham stimulation), we could not perform the second sequence in a reverse order. This could lead to a potential confound of block order. We, however, do not consider it as substantial since RT in sham sessions was not significantly different across the three locations. Finally, it should be noted that this is a single patient study based on a pathological brain with partial epilepsy and that the patient had a low IQ score. Therefore, a caution should be taken when interpreting the present results with respect to a normal healthy population.

By means of combined epicortical ERP recording and direct cortical stimulation, this study, for the first time, provided a direct piece of evidence that the pre-SMA, rostral to the NMA, actively engaged in the conflict-processing stream. Further case accumulation is warranted to establish the findings obtained in this single patient study. 


\section{Conflict of interests}

None declared.

\section{Acknowledgments}

This study was partly supported by Grants-in-Aid for Scientific

Research (C) 23591273 to R.M, and Grants-in-Aid for Scientific Research (C) 23591275 to A.I. from grant of Japan Ministry of Education, Culture, Sports, Science and Technology (MEXT). We wish to thank Dr. Michikazu Nakamura for referring the patient to us. 


\section{References}

Banich, M. T., Mackiewicz, K. L., Depue, B. E., Whitmer, A. J., Miller, G. A., \& Heller, W. (2009). Cognitive control mechanisms, emotion and memory: a neural perspective with implications for psychopathology. Neuroscience and biobehavioral reviews, 33, 613-630.

Barch, D. M., Braver, T. S., Akbudak, E., Conturo, T., Ollinger, J., \& Snyder, A. (2001). Anterior cingulate cortex and response conflict: effects of response modality and processing domain. Cerebral cortex, 11, 837-848.

Bartholow, B. D., Pearson, M. A., Dickter, C. L., Sher, K. J., Fabiani, M., \& Gratton, G. (2005). Strategic control and medial frontal negativity: beyond errors and response conflict. Psychophysiology, 42, 33-42.

Botvinick, M. M., Braver, T. S., Barch, D. M., Carter, C. S., \& Cohen, J. D. (2001). Conflict monitoring and cognitive control. Psychological review, 108, 624-652.

Botvinick, M. M., Cohen, J. D., \& Carter, C. S. (2004). Conflict monitoring and anterior cingulate cortex: an update. Trends in cognitive sciences, 8, 539-546.

Braver, T. S., Barch, D. M., Gray, J. R., Molfese, D. L., \& Snyder, A. (2001). Anterior cingulate cortex and response conflict: effects of frequency, inhibition and errors. Cerebral cortex, 11, 825-836.

Carter, C. S., Braver, T. S., Barch, D. M., Botvinick, M. M., Noll, D., \& Cohen, J. D. (1998). Anterior cingulate cortex, error detection, and the online monitoring of performance. Science, 280, 747-749.

Chen, C. Y., Muggleton, N. G., Tzeng, O. J. L., Hung, D. L., \& Juan, C. H. (2009). Control of prepotent responses by the superior medial frontal cortex. NeuroImage, 44, 537-545.

Cole, M. W., Yeung, N., Freiwald, W. A., \& Botvinick, M. (2009). Cingulate cortex: diverging data from humans and monkeys. Trends in neurosciences, 32, 566-574.

Eriksen, B. A., \& Eriksen, C.W. (1974). Effects of noise letters upon the identification of a target letter in a nonsearchtask. Perception \& Psychophysics, 16, 143-149.

Fellows, L. K., \& Farah, M. J. (2005). Is anterior cingulate cortex necessary for cognitive control? Brain, 128, 788-796.

Floden, D., \& Stuss, D. T. (2006). Inhibitory control is slowed in patients with right superior medial frontal damage. Journal of Cognitive Neuroscience, 18, 1843-1849.

Garavan, H., Ross, T. J., Murphy, K., Roche, R. A., \& Stein, E. A. (2002). Dissociable executive functions in the dynamic control of behavior: inhibition, error detection, and correction. NeuroImage, 17, 1820-1829.

Ikeda, A., Yazawa, S., Kunieda, T., Ohara, S., Terada, K., Mikuni, N., Nagamine, T., Taki, W., 
Kimura, J., \& Shibasaki, H. (1999). Cognitive motor control in human pre-supplementary motor area studied by subdural recording of discrimination/selection-related potentials. Brain, 122, 915-931.

Isoda, M., \& Hikosaka, O. (2007). Switching from automatic to controlled action by monkey medial frontal cortex. Nature neuroscience, 10, 240-248.

Kiehl, K. A., Liddle, P. F., \& Hopfinger, J. B. (2000). Error processing and the rostral anterior cingulate: an event-related fMRI study. Psychophysiology, 37, 216-223.

Kopp, B., Rist, F., \& Mattler, U. (1996). N200 in the flanker task as a neurobehavioral tool for investigating executive control. Psychophysiology, 33, 282-294.

Lüders, H. O., Dinner, D. S., Morris, H. H., Wyllie, E., \& Comair, Y. G. (1995). Cortical electrical stimulation in humans. The negative motor areas. Advances in neurology, $67,115-129$.

Lancaster, J. L., Tordesillas-Gutierrez, D., Martinez, M., Salinas, F., Evans, A., Zilles, K., Mazziotta, J. C., \& Fox, P. T. (2007). Bias between MNI and Talairach coordinates analyzed using the ICBM-152 brain template. Human brain mapping, 28, 1194-1205.

Lancaster, J. L., Woldorff, M. G., Parsons, L. M., Liotti, M., Freitas, C. S., Rainey, L., Kochunov, P. V., Nickerson, D., Mikiten, S. A., \& Fox, P. T. (2000). Automated Talairach atlas labels for functional brain mapping. Human brain mapping, 10, 120-131.

MacDonald, A. W., 3rd, Cohen, J. D., Stenger, V. A., \& Carter, C. S. (2000). Dissociating the role of the dorsolateral prefrontal and anterior cingulate cortex in cognitive control. Science, 288, 1835-1838.

Maris, E., \& Oostenveld, R. (2007). Nonparametric statistical testing of EEG- and MEG-data. Journal of neuroscience methods, 164, 177-190.

Matsumoto, R., Nair, D. R., Ikeda, A., Fumuro, T., Lapresto, E., Mikuni, N., Bingaman, W., Miyamoto, S., Fukuyama, H., Takahashi, R., Najm, I., Shibasaki, H., \& Lüders, H. O. (2011). Parieto-frontal network in humans studied by cortico-cortical evoked potential. Human brain mapping, doi: 10.1002/hbm.21407.

Matsumoto, R., Nair, D. R., LaPresto, E., Bingaman, W., Shibasaki, H., \& Luders, H. O. (2007). Functional connectivity in human cortical motor system: a cortico-cortical evoked potential study. Brain, 130, 181-197.

Matsumoto, R., Nair, D. R., LaPresto, E., Najm, I., Bingaman, W., Shibasaki, H., \& Lüders, H. O. (2004). Functional connectivity in the human language system: a cortico-cortical evoked potential study. Brain, 127, 2316-2330.

Milham, M. P., \& Banich, M. T. (2005). Anterior cingulate cortex: an fMRI analysis of conflict 
specificity and functional differentiation. Human brain mapping, 25, 328-335.

Milham, M. P., Banich, M. T., Webb, A., Barad, V., Cohen, N. J., Wszalek, T., \& Kramer, A. F. (2001). The relative involvement of anterior cingulate and prefrontal cortex in attentional control depends on nature of conflict. Brain research. Cognitive brain research, 12, 467-473.

Nachev, P., Kennard, C., \& Husain, M. (2008). Functional role of the supplementary and pre-supplementary motor areas. Nature reviews. Neuroscience, 9, 856-869.

Ridderinkhof, K. R., Ullsperger, M., Crone, E. A., \& Nieuwenhuis, S. (2004). The role of the medial frontal cortex in cognitive control. Science, 306, 443-447.

Rossi, S., Hallett, M., Rossini, P. M., \& Pascual-Leone, A. (2009). Safety, ethical considerations, and application guidelines for the use of transcranial magnetic stimulation in clinical practice and research. Clinical neurophysiology, 120, 2008-2039.

Rushworth, M. F., Walton, M. E., Kennerley, S. W., \& Bannerman, D. M. (2004). Action sets and decisions in the medial frontal cortex. Trends in cognitive sciences, 8, 410-417.

Rusnáková, Š., Daniel, P., Chladek, J., Jurák, P., \& Rektor, I. (2011). The Executive Functions in Frontal and Temporal Lobes: A Flanker Task Intracerebral Recording Study. Journal of clinical neurophysiology, 28, 30-35.

Sharp, D. J., Bonnelle, V., De Boissezon, X., Beckmann, C. F., James, S. G., Patel, M. C., \& Mehta, M. A. (2010). Distinct frontal systems for response inhibition, attentional capture, and error processing. Proceedings of the National Academy of Sciences of the United States of America, 107, 6106-6111.

Sheth, S. A., Mian, M. K., Patel, S. R., Asaad, W. F., Williams, Z. M., Dougherty, D. D., Bush, G., \& Eskandar, E. N. (2012). Human dorsal anterior cingulate cortex neurons mediate ongoing behavioural adaptation. Nature, 488, 218-221.

Simmonds, D. J., Pekar, J. J., \& Mostofsky, S. H. (2008). Meta-analysis of Go/No-go tasks demonstrating that fMRI activation associated with response inhibition is task-dependent. Neuropsychologia, 46, 224-232.

Taylor, P. C. J., Nobre, A. C., \& Rushworth, M. F. S. (2007). Subsecond changes in top-down control exerted by human medial frontal cortex during conflict and action selection: a combined transcranial magnetic stimulation- electroencephalography study. The Journal of neuroscience, 27, 11343-11353.

Ullsperger, M., \& von Cramon, D. Y. (2001). Subprocesses of performance monitoring: a dissociation of error processing and response competition revealed by event-related fMRI and ERPs. NeuroImage, 14, 1387-1401.

Ullsperger, M., \& von Cramon, D. Y. (2003). Error monitoring using external feedback: 
specific roles of the habenular complex, the reward system, and the cingulate motor area revealed by functional magnetic resonance imaging. The Journal of neuroscience, 23, 4308-4314.

van Veen, V., \& Carter, C. S. (2002a). The anterior cingulate as a conflict monitor: fMRI and ERP studies. Physiology \& behavior, 77, 477-482.

Van Veen, V., \& Carter, C. S. (2002b). The timing of action-monitoring processes in the anterior cingulate cortex. Journal of Cognitive Neuroscience, 14, 593-602.

Verfaellie, M., \& Heilman, K. M. (1987). Response preparation and response inhibition after lesions of the medial frontal lobe. Archives of neurology, 44, 1265-1271.

Yeung, N., Botvinick, M. M., \& Cohen, J. D. (2004). The neural basis of error detection: conflict monitoring and the error-related negativity. Psychological review, 111, 931-959. 
Fig. 1. Experimental design. (a) Three stimulus sites [1. the inferior part of the medial superior frontal gyrus (inferior medial SFG), 2. the superior part of the medial SFG (superior medial SFG), and 3. the middle frontal gyrus (MFG)] are shown in the preoperative 3D brain MRI co-registered with subdural electrodes. The electrodes corresponding to the supplementary negative motor area (NMA) are also shown. The epileptogenic lesion (tumor) was located in the supplementary motor area (SMA). (b) A schema of the experimental paradigm. During presentation of the flanker-signal, either sham or real $(50 \mathrm{~Hz}, 5 \mathrm{~mA}$ for $1 \mathrm{~s})$ stimulation was applied to the three stimulus sites. VAC: vertical anterior commissure line, usually representing a border between the pre-SMA and the SMA proper. VPC: vertical posterior commissure line. 
Fig. 2. ERPs recorded during the sessions of sham stimulation. Congruent $(\mathrm{n}=71$, gray $)$ and incongruent $(\mathrm{n}=72$, black $)$ trials are shown separately. The left schema indicates location of the recording electrodes. First, ERPs of the two conditions were compared during the flanker-signal presentation (shaded in light gray) at each sampling point using two-tailed two sample $t$ test. The black bars under the figures during this time segment indicate $p<$ $0.05(|t(141)|>1.977)$. Then, statistical analysis at the cluster levels was performed. Only the cluster highlighted by the thinner bar and the arrowhead (350-886 ms after onset of the flanker-signal) at the inferior medial SFG stimulus site (electrode with an asterisk) showed significance ( $p$ $=0.02$, nonparametric permutation test) between the two conditions. The other conventions are the same as for Fig. 1. 
Fig. 3. Reaction time (RT) at the three stimulus sites (inferior medial SFG, superior medial SFG, MFG). RT was shown separately for each set [2 (congruent: $\mathrm{C}$ or incongruent: I) $\times 2$ (real stimulation: stim or sham stimulation: sham)]. RT in each set was judged to be based on a standard normal distribution ( $p>0.05$, Kolmogorov-Smirnov test). Two sample $t$ test was performed in order to evaluate the RT difference between sham and real stimulation for the two conditions [congruent, incongruent; $p<0.025$ (Bonferroni corrected)] at each stimulus site. ${ }^{*} t=2.494, p=0.016$. n.s: not statistically significant ( $p>0.025$ ). Error bars represent SEM. $\mathrm{n}$ : the number of trials in each set. 
Fig. 4. A comparison between the present invasive findings and non-invasive fMRI studies in the MNI standard space about conflict processing. The electrode locations (filled circle) and peak loci of previous activation studies (filled diamond) were put together onto a representative brain slice at $4 \mathrm{~mm}$ from midline: red (inferior medial SFG, present study), yellow (NMA, present study), orange (Carter, et al., 1998), white (MacDonald, et al., 2000), light-green (Barch, et al., 2001), burlywood (Braver, et al., 2001), dark-blue (Milham, et al., 2001), blue (Ullsperger \& von Cramon, 2001), light-blue (Ullsperger \& von Cramon, 2003), magenta (Milham \& Banich, 2005). Talairach coordinates were converted to MNI coordinates by icbm2tal transform (http://brainmap.org/icbm2tal/) (Lancaster, et al., 2007). 


\section{Fig. 1.}

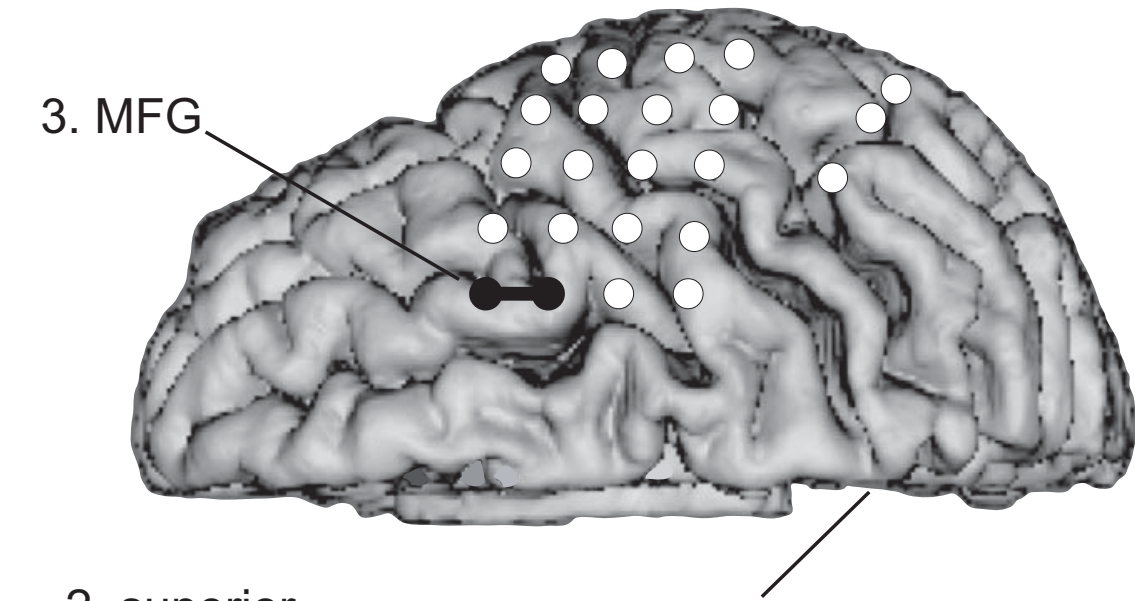

2. superior medial SFG

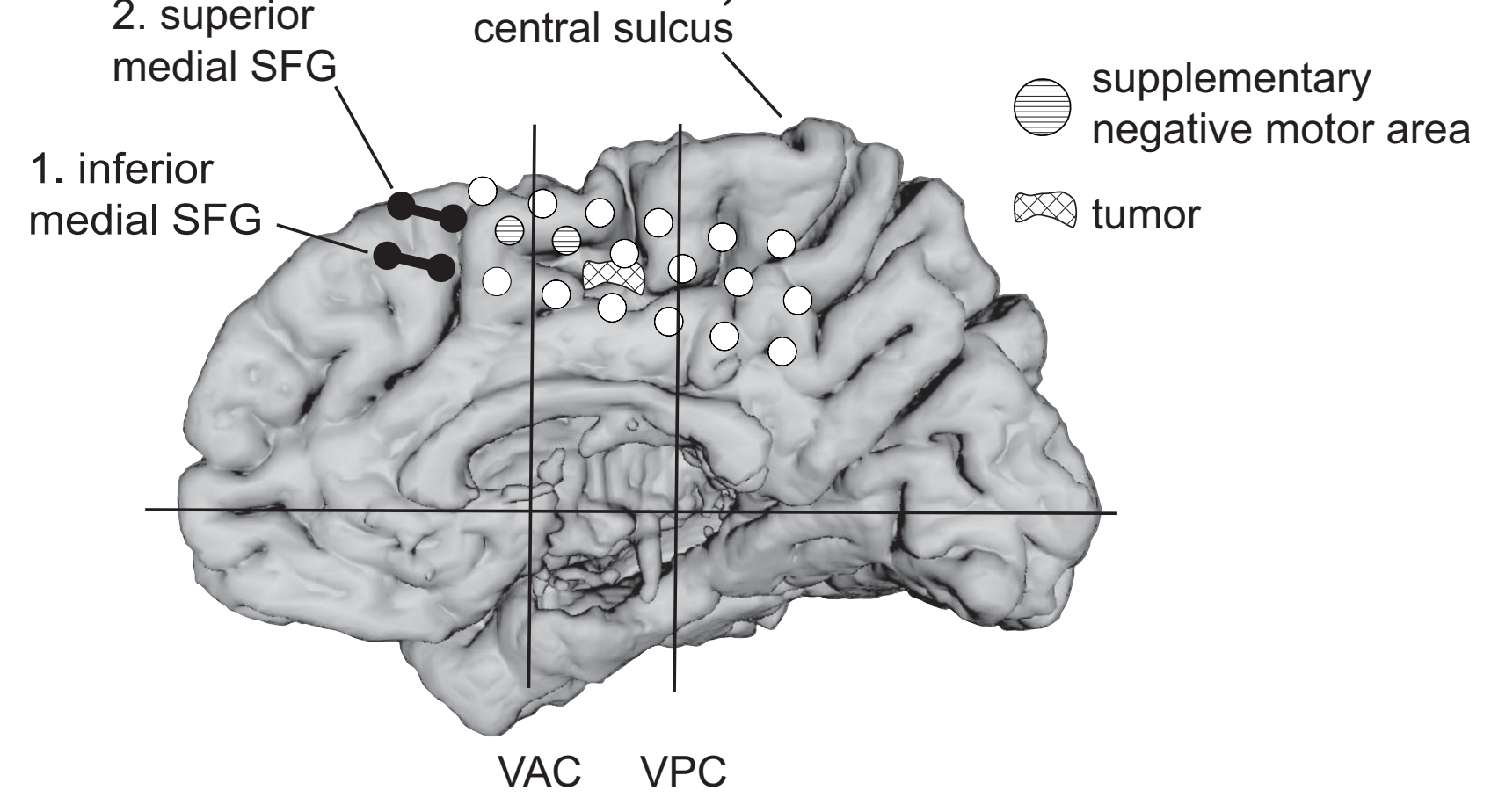

O

warning

direct cortical

stimulation

or sham

stimulation at 


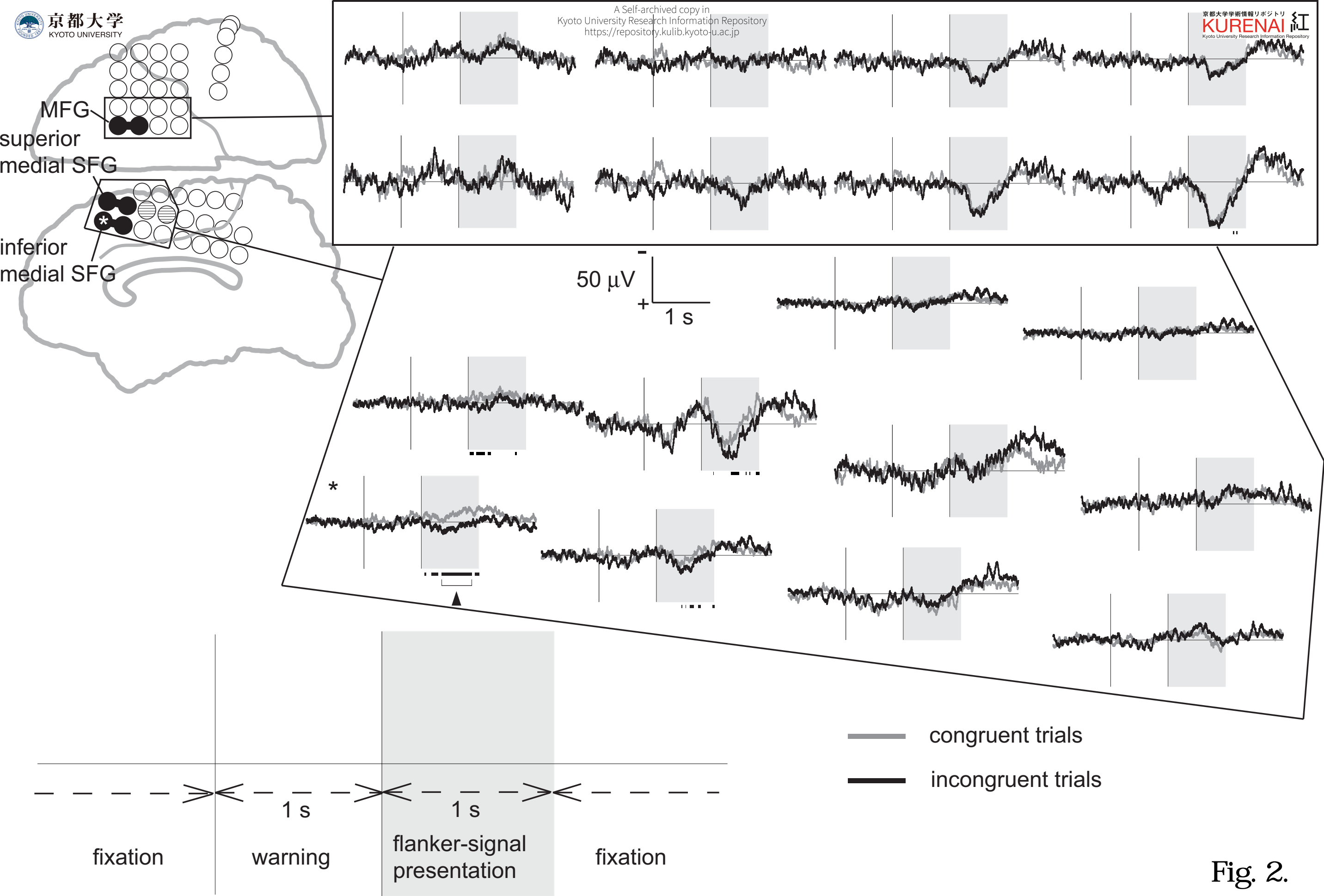




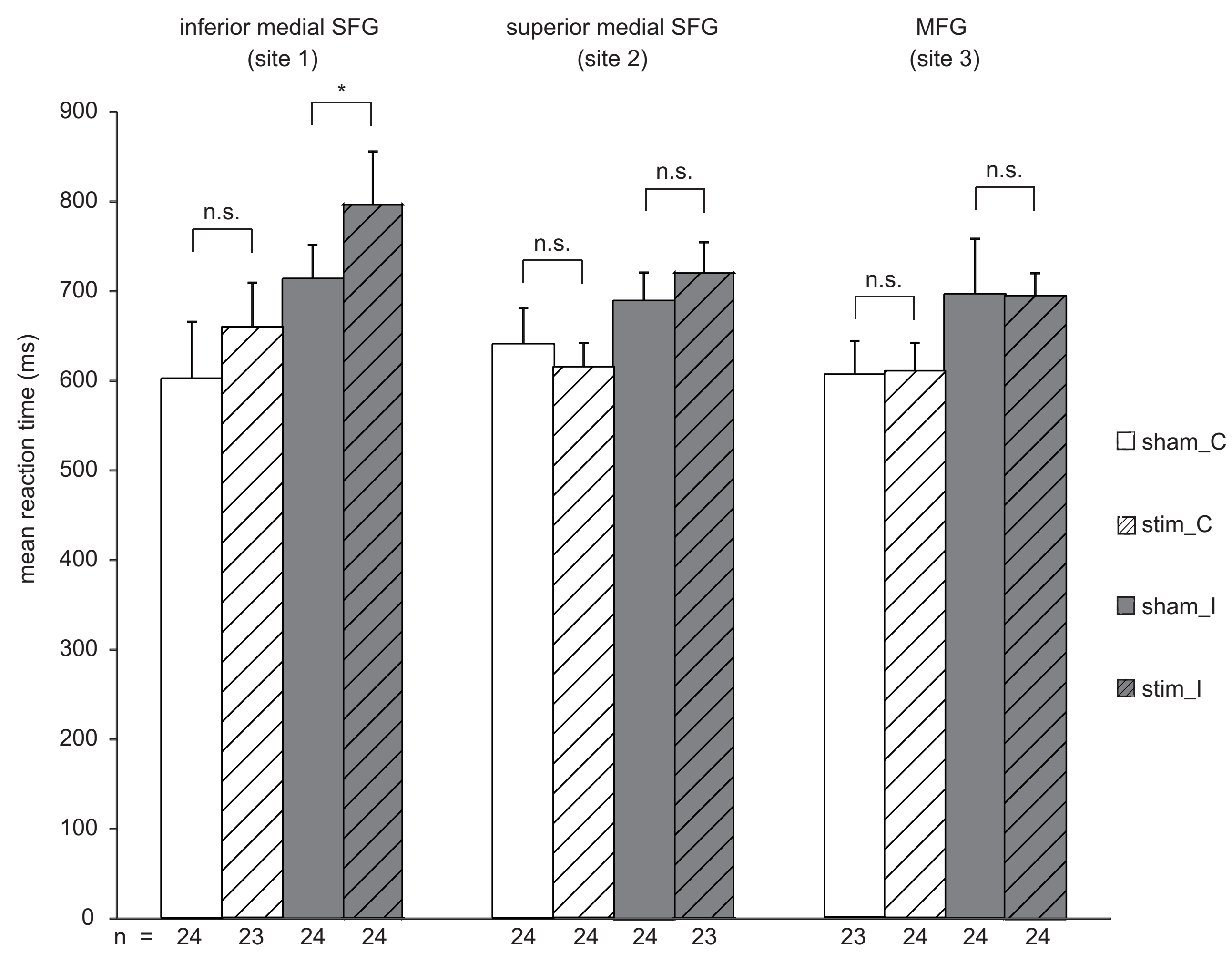

Fig. 3. 


\section{Supplementary Table}

Reaction time (mean \pm SEM ms) in the modified flanker task

\section{Stimulus sites}

The type of set

inferior medial SFG

superior medial SFG

MFG

Sham_C

$600 \pm 66(\mathrm{n}=24)$

$639 \pm 43(\mathrm{n}=24)$

$606 \pm 39(\mathrm{n}=23$, blink; 1$)$

Stim_C

$658 \pm 51(\mathrm{n}=23$, error; 1$)$

$613 \pm 28(\mathrm{n}=24)$

$609 \pm 33(\mathrm{n}=24)$

Sham_I

$711 \pm 40(\mathrm{n}=24)$

$688 \pm 32(\mathrm{n}=24)$

$694 \pm 64(\mathrm{n}=24)$

Stim_I

$797 \pm 59(\mathrm{n}=24)$

$721 \pm 33(\mathrm{n}=23$, blink; 1$)$

$694 \pm 25(\mathrm{n}=24)$

SFG: superior frontal gyrus, MFG: middle frontal gyrus

C: congruent, I: incongruent, Stim: direct electrical cortical stimulation

$\mathrm{n}$ : the number of trials in each set 